\title{
Relações Entre Crenças, Atitudes e Saúde Mental de Idosos na Pandemia da Covid-19
}

\author{
Relations Between Beliefs, Attitudes and Mental Health in Elderly People \\ During the Covid-19 Pandemic
}
Relaciones Entre Creencias, Actitudes y Salud Mental de los Ancianos en la Pandemia de Covid-19

\author{
Heloísa Gonçalves Ferreira ${ }^{1}$ \\ Universidade do Estado do Rio de Janeiro (UERJ)
}

\begin{abstract}
Resumo
Contextualização do tema e problema: A saúde mental de idosos encontra-se em risco durante pandemia da covid-19. Características da amostra: 384 idosos usuários da Internet. Instrumentos: Questionário (informações sociodemográficas, crenças/atitudes sobre covid-19), PHQ-2 para avaliar depressão, versão curta UCLA-BR para aferir solidão, escala de Suporte Social e Escala Breve de Resiliência. Procedimento: Idosos responderam on-line aos instrumentos para investigar frequências e relações entre indicadores de saúde mental e crenças/atitudes. Resultados: 15,1\% apresentaram triagem positiva para depressão. Autoconfiança para realizar isolamento domiciliar relacionou-se significativamente com menor frequência de saídas de casa e melhores índices de saúde mental. Piores índices de saúde mental associaram-se a uma percepção mais forte sobre a ameaça da covid-19 à saúde. Discussão: A autoconfiança em realizar isolamento domiciliar e as crenças sobre a gravidade e vulnerabilidade à doença são componentes psicológicos relevantes de serem trabalhados em intervenções para promoção da saúde mental e maior adesão ao isolamento domiciliar. Palavras-chave: idosos, depressão, resiliência, isolamento social, coronavírus
\end{abstract}

\begin{abstract}
Research problem: Elderly's mental health is at risk during covid-19 pandemic. Sample's characteristics: 384 elderly people who are Internet users. Instruments: questionnaire to access sociodemographic data and beliefs/attitudes about covid-19, PHQ-2 to evaluate depression, UCLA-BR short version to evaluate loneliness, Social Support Scale, and the Brief Resilience Scale. Procedure: participants answered an online survey in order to investigate frequencies and relations between mental health indicators and beliefs/attitudes about covid-19. Results: 15.1\% showed positive screening for depression. Self-confidence for home isolation showed significant relations with lower frequency of leaving the house and better mental health. Worse mental health was associated to a stronger perception of covid-19 threat for health. Discussion: Self-confidence for home isolation and beliefs regarding covid-19 severity and vulnerability might be relevant psychological components to be target in interventions to ensure greater adherence to home isolation and better mental health.
\end{abstract}

Keywords: elderly, depression, resilience, social isolation, coronavirus

\section{Resumen}

Contextualización del tema y del problema: la salud mental de los ancianos está en riesgo durante la pandemia de covid-19. Características de la muestra: 384 ancianos que son usuarios de internet. Instrumentos: cuestionario (información sociodemográfica, creencias / actitudes sobre covid-19), PHQ-2 para evaluar la depresión, versión corta del UCLA-BR para medir la soledad, Escala de Apoyo social y Escala Breve de Resiliencia. Procedimiento: los ancianos respondieron al cuestionario online para investigar frecuencias y relaciones entre indicadores de salud mental y creencias/actitudes. Resultados: el 15.1\% tuvo un resultado positivo de depresión. La autoconfianza para realizar el aislamiento en el hogar se relacionó significativamente con menor frecuencia de abandono del hogar y mejores índices de salud mental. Peor salud mental se asoció a una percepción más fuerte de amenaza del covid-19 en relación a su salud. Discusión: La autoconfianza en la realización del aislamiento en el hogar y las creencias sobre la gravedad y la vulnerabilidad a la enfermedad son componentes psicológicos relevantes para trabajar en las intervenciones para promover la salud mental y una mayor adherencia al aislamiento en el hogar.

Palabras clave: ancianos, depresión, resiliencia, aislamiento social, coronavirus

\footnotetext{
${ }^{1}$ Endereço para contato: Rua São Francisco Xavier, 524, Maracanã, Rio de Janeiro, RJ, Brasil, CEP: 20550-900. Telefone: (21) 2334-0000. E-mail: helogf@gmail.com
} 


\section{Introdução}

Em dezembro de 2019, foram documentados os primeiros casos de quadros de pneumonia causados pelo novo coronavírus (SARS-CoV-2) em Wuhan, na China (Deng \& Peng, 2020). A nova doença foi nomeada pela Organização Mundial de Saúde (OMS) como covid-19 e se espalhou rapidamente pela China e pelo globo, sendo caracterizada como uma pandemia (World Health Organization, 2020a). Até 11 de junho de 2020, a OMS contabilizou 7.273.958 casos confirmados da doença, com 413.372 mortes registradas no mundo, sendo que, no Brasil, até então, haviam sido reportados 739.503 casos, com 38.406 mortes (World Health Organization, 2020b). Idosos com doenças crônicas, tais como diabetes, hipertensão e doenças cardiovasculares, estão entre os grupos de risco para a covid-19, uma vez que indivíduos com essas caraterísticas apresentam maior comprometimento da função imunológica (Liu, Chen, Lin \& Han, 2020). A taxa de letalidade pode chegar a quase 15\% em pacientes com 80 anos ou mais (Brasil, 2020).

Em situações de pandemia, como é o caso da covid-19, e na ausência de vacinas e tratamentos específicos disponíveis para lidar com o vírus, ações tradicionais não farmacológicas de saúde pública são empregadas com o objetivo de conter a propagação do vírus e evitar novos casos de contaminação e mortes. O isolamento, a quarentena, o distanciamento social e a contenção comunitária são exemplos dessas ações tradicionais que visam prevenir a transmissão pela separação e afastamento entre as pessoas, sendo que todas essas medidas envolvem restrição de contato social (Wilder-Smith \& Freedman, 2020).

No caso de idosos, além de constituírem população de risco para a infecção por coronavírus (Liu et al., 2020), podem ser seriamente afetados pelas medidas de restrição social, uma vez que esta população já é mais susceptível ao desenvolvimento de sentimentos de solidão e transtornos mentais, tais como a depressão, tendo o isolamento social como importante fator de risco para esses desfechos (Armitage \& Nellums, 2020). Ainda, considerando tantas privações que a medida acarreta (restrição de contato familiar e de amigos, restrição de atividades etc.), muitos idosos podem apresentar resistência em seguir essas medidas à risca, colocando a saúde seriamente em risco.

Desta forma, os padrões de comportamentos, crenças e as relações interpessoais dos indivíduos influenciam significativamente os processos de saúde e doença (Borges \& Barletta, 2015). O Modelo de Crenças em Saúde (MCS) proposto por Rosenstock, Stretcher e Becker $(1988,1994)$ ajuda a compreender como um indivíduo organiza suas crenças, percepções, atitudes e intenções de forma a predizer cuidados preventivos de saúde. Segundo o MCS, a adoção de padrões de comportamentos promotores de saúde vincula-se à inter-relação de três condições: (1) interesse e reconhecimento do indivíduo sobre ações importantes e necessárias à prevenção de sua saúde; (2) percepção do indivíduo como vulnerável à doença e ciência das consequências caso a doença seja contraída; e (3) reconhecimento de que os novos padrões comportamentais são efetivos para diminuir riscos da doença e que os benefícios relacionados a adoção de novos padrões comportamentais favorecedores da saúde superam qualquer custo em realizá-los (Borges \& Barletta, 2015).

O MCS assume que um padrão de comportamento preventivo à saúde (no caso, realizar o isolamento domiciliar) tem relação com o indivíduo se considerar vulnerável à doença (vulnerabilidade percebida) e perceber a gravidade das consequências caso a doença seja 
contraída (gravidade percebida); da mesma forma, tal comportamento relaciona-se também à crença de que o comportamento adotado ajuda a prevenir a doença (benefícios percebidos), mesmo que tal comportamento implique restrições, obstáculos e desconfortos (barreiras percebidas), sendo ainda influenciado pelo quanto o indivíduo se avalia capaz (autoconfiança percebida) de adotar o padrão de comportamento recomendado (Rosenstock et al., 1988,1994; Borges \& Barletta, 2015).

Portanto, o MCS parece ser uma opção teórica interessante a fim de elucidar as percepções e atitudes dos indivíduos idosos com relação às medidas preventivas em saúde para o combate da infecção pelo coronavírus, que incluem o isolamento social. Por se tratar de uma crise até então sem precedentes no contexto brasileiro, a literatura nacional não conta ainda com informações sobre como os idosos brasileiros estão lidando com esta situação de crise e quais padrões de crenças e atitudes emergem diante do enfrentamento à pandemia. Além disso, a questão do isolamento social, por um lado, parece ser crucial para prevenir que idosos contraiam uma doença que pode ser fatal, mas, por outro lado, representa uma medida de alto custo social que resulta em grandes privações e até mesmo maior probabilidade de desenvolvimento de sentimentos de solidão e depressão, principalmente se o idoso contar com pouca disponibilidade de suporte social e poucos recursos psicológicos de enfrentamento. Sendo assim, o mapeamento das crenças, atitudes e condições de saúde mental no contexto da pandemia da covid-19 é imprescindível para o desenvolvimento de intervenções que visam promover comportamentos favorecedores da saúde da população idosa. Análises desta natureza podem contribuir ainda para a previsão e alteração de comportamentos de risco em potencial, favorecendo o desenvolvimento de repertórios mais adequados que protegem a saúde de grupos e indivíduos mais vulneráveis.

O objetivo geral do presente estudo foi investigar a frequência e as relações entre indicadores de saúde mental, crenças e atitudes de idosos ante a pandemia da covid-19. Os objetivos específicos foram: (1) descrever a frequência de crenças e atitudes sobre o Coronavírus e isolamento domiciliar; (2) descrever níveis de depressão, solidão, resiliência e suporte social em idosos durante o período da pandemia da covid-19; (3) investigar as relações entre as crenças e atitudes sobre o coronavírus e o isolamento domiciliar; e (4) investigar as relações entre crenças/atitudes sobre o coronavírus/isolamento domiciliar e indicadores de saúde mental.

\section{Método}

\section{Participantes}

O presente estudo foi caracterizado por amostra de conveniência, estruturada a partir de coleta on-line com idosos que tinham acesso à Internet. Acessaram os instrumentos deste estudo um total de 422 pessoas. Cinco pessoas declararam não querer participar da pesquisa após leitura do Termo de Consentimento Livre e Esclarecido e, por isso, não responderam aos instrumentos. Trinta e três pessoas declararam ter menos de 60 anos e, por isso, foram excluídas da amostra. A amostra final foi composta por 384 indivíduos com 60 anos ou mais, que aceitaram participar do estudo e responderam on-line, de forma completa, os instrumentos da pesquisa. A coleta on-line se deveu às dificuldades e limitações de contato presencial que o momento histórico impõe. 


\section{Instrumentos}

Questionário sociodemográfico contendo informações sobre sexo, escolaridade, estado civil, com quantas pessoas o idoso mora, se apresenta doenças crônicas e outras informações para traçar perfil sociodemográfico da amostra.

Crenças e atitudes sobre o coronavírus e o isolamento domiciliar: o isolamento domiciliar foi aferido por meio de uma única pergunta ("Nas últimas duas semanas, quantas vezes você saiu de casa?"), com quatro opções de resposta: nenhuma vez; de uma a cinco vezes; de seis a dez vezes; e mais de dez vezes. Também foi questionado o quão vulnerável o idoso se sentia ante a possibilidade de contrair covid-19; o quão grave ele acreditava que poderiam ser as consequências da doença; o quanto concordava que o isolamento domiciliar poderia ajudar a diminuir a propagação e as chances de contrair o coronavírus; e o quanto o idoso se sentia capaz de realizar o isolamento domiciliar. Todas essas perguntas foram elaboradas tendo por base os pressupostos principais do MCS (Rosenstock et al., 1988, 1994) e foram aferidas por uma escala Likert de quatro pontos.

A depressão foi aferida pela PHQ-2 (Patient Health Questionnaire - versão de dois itens), derivada da PHQ-9 e baseada em critérios discriminativos para episódios de depressão maior, referentes à anedonia e ao humor deprimido. A escala é composta por dois itens que fazem referência aos 14 dias anteriores a sua aplicação, com respostas a partir de uma escala Likert de quatro pontos que varia de zero (nunca) a três (quase todos os dias). Escores iguais ou maiores que três são indicativos de depressão (Gaya, 2011). As propriedades psicométricas da escala para uso com a população brasileira foram investigadas por Osório, Mendes, Crippa e Loureiro (2009), Gaya (2011) e Chagas et al. (2011), e os estudos no geral demonstram que a escala é válida para uso na população brasileira para triar sintomas de depressão. No presente estudo, a escala apresentou consistência interna igual a 0,73.

UCLA-BR-versão curta: esta foi derivada a partir da UCLA-BR, a qual é a versão brasileira da UCLA-R (Russel, Peplau, \& Cutrona, 1980), que avalia solidão em adultos e idosos (Barroso, Andrade, Midgett, \& Carvalho, 2016). Em sua versão curta, a escala conta com seis afirmações que descrevem os estados afetivos e cognitivos da solidão, em que o respondente tem de assinalar a frequência (que varia de 0-nunca a 3-frequentemente) com que se identifica com cada afirmação ultimamente. A versão curta da UCLA-BR apresentou boa consistência interna $(\alpha=0,87)$ e relações significativas com depressão e suporte social. Na presente amostra, foi obtida consistência interna igual a 0,82.

O suporte social percebido será aferido a partir de quatro perguntas ("O/a senhor/a diria que tem várias pessoas com quem conversar ao se sentir sozinho?"; "O/a senhor/a teria facilidade em encontrar pessoas que possam te ajudar nos seus afazeres se estiver doente?"; "O/a senhor/a tem com quem contar quando precisa de uma sugestão de como lidar com um problema?"; "O/a senhor/a tem pelo menos uma pessoa cuja opinião você confia plenamente?"), com opções de respostas em escala Likert de quatro pontos, variando de "nunca" a "sempre". As referidas perguntas foram retiradas do protocolo original de pesquisa do Estudo Fibra, o qual avaliou o suporte social percebido em idosos brasileiros (Neri \& Vieira, 2013), e analisam, especificamente, a disponibilidade de suporte socioemocional, instrumental, informativo e afetivo. Para a presente amostra, o instrumento apresentou consistência interna igual a 0,74. 
A resiliência foi aferida a partir da Escala Breve de Resiliência, adaptada para uso no Brasil a partir da versão original desenvolvida por Smith et al. (2008). A referida versão conta com cinco itens, com opções de resposta a partir de escala Likert de cinco pontos, que varia de "discordo fortemente" até "concordo fortemente". Para a presente amostra a consistência interna foi igual a 0,81 . Os escores da escala variam de 5 a 25 e escores maiores indicam níveis maiores de resiliência.

\section{Procedimento de Coleta de dados}

Todos os itens dos instrumentos da pesquisa foram organizados em formulário Google Forms, para possibilitar acesso on-line dos participantes da pesquisa. Foram enviados convites on-line (via e-mail, WhatsApp, Facebook, Instagram etc.), com o link para acesso ao protocolo de pesquisa, a pessoas com 60 anos ou mais. 0 indivíduo com 60 anos ou mais que se voluntariasse a participar da pesquisa acessava o link para resposta aos instrumentos. Primeiramente, o participante tinha acesso ao Termo de Consentimento Livre e Esclarecido (TCLE). Após leitura do TCLE, o participante era questionado se compreendia a natureza do estudo e se concordava em participar. Se o participante assinalasse a opção "compreendi a natureza do presente estudo e concordo em participar da pesquisa", era então direcionado para responder aos instrumentos da pesquisa. A coleta on-line ocorreu durante o mês de abril e maio de 2020.

\section{Procedimento de Análise de Dados}

Os dados foram analisados segundo técnicas de estatísticas descritivas (média, desvio-padrão, valores mínimos e máximos, frequências etc.) e inferenciais (testes de correlação) apropriadas à natureza das variáveis. Foi verificada a distribuição das variáveis, sendo constatado que as variáveis não apresentavam distribuição normal. Por esta razão, adotou-se o coeficiente de correlação de Spearman para as análises de correlação. Os dados foram analisados utilizando-se o Microsoft Excel e o software de análises estatísticas IBM SPSS 20.0. Valores de $p$ menores ou iguais a 0,05 foram considerados significativos.

\section{Aspectos Éticos}

O desenvolvimento deste estudo está amparado nas resoluções n. 466, de 12/12/2012, e n. 510, de 07/04/2016. Foi aprovado pelo CEP do Hospital Universitário Pedro Ernesto vinculado à Universidade do Estado do Rio de Janeiro, com CAAE 30915820.2.0000.5259.

\section{Resultados}

A Tabela 1 exibe o perfil sociodemográfico da amostra. A maioria era do sexo feminino (66,9\%), tinha entre 60 e 70 anos (79,4\%), tinha mais de 8 anos de estudo (93,5\%), era casada $(53,4 \%)$, proveniente da região Sudeste brasileira $(84,4 \%)$, aposentada $(77,3 \%)$ e sem trabalhar $(56,5 \%)$, com alguma doença crônica $(51,3 \%)$ e morando com outras pessoas (77,9\%). 
Tabela 1

Perfil Sociodemográfico da amostra

\begin{tabular}{|c|c|c|c|}
\hline Variáveis & Categorias & $\%$ & n. \\
\hline \multirow{2}{*}{ Sexo } & Feminino & 66,9 & 257 \\
\hline & Masculino & 33,1 & 127 \\
\hline \multirow{3}{*}{ Idade } & $60-70$ & 79,4 & 305 \\
\hline & $71-80$ & 18,3 & 70 \\
\hline & $80+$ & 2,3 & 9 \\
\hline \multirow{3}{*}{ Anos de estudo } & Não estudou & 0,8 & 3 \\
\hline & Até 8 anos de estudo & 5,7 & 22 \\
\hline & Mais que 8 anos de estudo & 93,5 & 359 \\
\hline \multirow{4}{*}{ Estado civil } & Solteiro & 11,5 & 44 \\
\hline & Casado & 53,4 & 205 \\
\hline & Divorciado & 20,1 & 77 \\
\hline & Viúvo & 15,1 & 58 \\
\hline \multirow{4}{*}{ Região Brasileira } & Sudeste & 84,4 & 324 \\
\hline & Sul & 3,6 & 14 \\
\hline & Centro-Oeste/Norte & 1,6 & 6 \\
\hline & Nordeste & 10,5 & 40 \\
\hline \multirow{2}{*}{ Aposentado/pensionista } & Sim & 77,3 & 297 \\
\hline & Não & 22,7 & 87 \\
\hline \multirow{2}{*}{ Trabalha atualmente } & Sim & 43,5 & 167 \\
\hline & Não & 56,5 & 217 \\
\hline \multirow{2}{*}{ Apresenta alguma doença crônica } & Sim & 51,3 & 197 \\
\hline & Não & 48,7 & 187 \\
\hline \multirow{4}{*}{ Arranjo domiciliar } & Mora sozinho & 22,1 & 85 \\
\hline & Mora com cônjuge & 27,6 & 106 \\
\hline & Mora com cônjuge e filhos & 22,4 & 86 \\
\hline & Mora com outros familiares/amigos & 27,9 & 107 \\
\hline
\end{tabular}

Os descritivos para as crenças e atitudes sobre o coronavírus/isolamento domiciliar são exibidos na Tabela 2. Observa-se que 31,3\% da amostra relatou não sair nenhuma vez de casa, ao passo que a maioria $(56,3 \%)$ relatou sair de casa de uma a cinco vezes num período de duas semanas de pandemia. Uma proporção maior da amostra relatou perceber maior gravidade $(74,5 \%)$ do que maior vulnerabilidade ao vírus (57,6\%). A maioria dos participantes concordava que o isolamento poderia diminuir a propagação do vírus $(92,5 \%)$ e evitar contaminação (93\%). Ainda, 95,1\% da amostra relatou se sentir capaz de realizar o isolamento domiciliar. 
Tabela 2

Frequência de crenças e atitudes sobre covid-19 e isolamento domiciliar

\begin{tabular}{llcc}
\hline Variáveis & Categorias & \% & N. \\
\hline \multirow{2}{*}{$\begin{array}{l}\text { Frequência de saídas de casa no } \\
\text { intervalo de duas semanas }\end{array}$} & Nenhuma vez & 31,3 & 120 \\
& De uma a cinco vezes & 56,3 & 216 \\
Percepção de vulnerabilidade para & Nais de cinco vezes & 12,5 & 48 \\
contrair coronavírus & Vulnerável/Extremamente vulnerável & 57,6 & 221 \\
Percepção de gravidade das & Nada grave/Pouco grave & 25,5 & 98 \\
consequências da covid-19 & Grave/Extremamente grave & 74,5 & 286 \\
Isolamento domiciliar ajuda a & Discordo fortemente/Discordo & 7,5 & 29 \\
diminuir propagação do coronavírus & Concordo/Concordo fortemente & 92,5 & 355 \\
Isolamento domiciliar ajuda a evitar & Discordo fortemente/Discordo & 7 & 27 \\
contaminação pelo coronavírus & Concordo/Concordo fortemente & 93 & 357 \\
Eu me sinto capaz de realizar & Discordo fortemente/Discordo & 4,9 & 19 \\
isolamento domiciliar & Concordo/Concordo fortemente & 95,1 & 365 \\
\hline
\end{tabular}

Os descritivos dos indicadores de saúde mental encontram-se na Tabela 3. Foi constatado que $15,1 \%$ da amostra apresentou triagem positiva para depressão. As médias indicam que, no geral, a amostra apresentou índices mais altos para suporte social e resiliência, e baixos índices de solidão e depressão.

Tabela 3

Descritivos indicadores de saúde mental

\begin{tabular}{ccccc}
\hline Variáveis & Média & Desvio-Padrão & Mínimo & Máximo \\
\hline Solidão & 5,15 & 3,86 & 0 & 18 \\
Suporte Social & 13,65 & 2,35 & 6 & 16 \\
Resiliência & 18,7 & 4,15 & 5 & 25 \\
Depressão & 1,3 & 1,46 & 0 & 6 \\
Depressão & \multicolumn{2}{c}{ Com sintomas } & \multicolumn{2}{c}{$15,1 \%$} \\
& \multicolumn{2}{c}{ Sem sintomas } \\
\hline
\end{tabular}

A Tabela 4 exibe as relações entre as crenças e atitudes sobre o coronavírus/isolamento domiciliar. Foram encontradas relações significativas de magnitudes fracas e moderadas entre quase todos os construtos. A frequência das saídas de casa não apresentou associação significativa com percepção de vulnerabilidade à covid-19 e autoconfiança para realizar isolamento domiciliar. 
Tabela 4

Relações entre crenças e atitudes sobre covid-19 e isolamento domiciliar

\begin{tabular}{|c|c|c|c|c|c|}
\hline & $\begin{array}{c}\text { Percepção } \\
\text { vulnerabilidade }\end{array}$ & $\begin{array}{l}\text { Percepção } \\
\text { gravidade }\end{array}$ & $\begin{array}{l}\text { Isolamento } \\
\text { diminui } \\
\text { propagação }\end{array}$ & $\begin{array}{c}\text { Isolamento } \\
\text { evita } \\
\text { contaminação }\end{array}$ & $\begin{array}{c}\text { Autoconfiança } \\
\text { para isolamento } \\
\text { domiciliar }\end{array}$ \\
\hline $\begin{array}{l}\text { Frequência de saídas } \\
\text { de casa }\end{array}$ & -.075 & $-.209^{* *}$ & $-.257^{* *}$ &,$- 188^{* *}$ & $-.352^{* *}$ \\
\hline $\begin{array}{l}\text { Percepção } \\
\text { vulnerabilidade }\end{array}$ & - & $.545^{* *}$ & $.164^{* *}$ &, $203^{* *}$ & .079 \\
\hline Percepção gravidade & - & - & $.262^{* *}$ &, $270^{* *}$ & $.147^{* *}$ \\
\hline $\begin{array}{l}\text { Isolamento diminui } \\
\text { propagação }\end{array}$ & - & - & - & $.682^{* *}$ & $.412^{* *}$ \\
\hline $\begin{array}{l}\text { Isolamento evita } \\
\text { contaminação }\end{array}$ & - & - & - & - & $.375^{* *}$ \\
\hline
\end{tabular}

As relações entre crenças/atitudes sobre coronavírus/isolamento domiciliar e os indicadores de saúde mental podem ser visualizadas na Tabela 5. Foram encontradas relações estatisticamente significativas de magnitude fraca entre as crenças e os indicadores de saúde mental, que revelam que uma maior frequência de sintomas depressivos está relacionada a uma menor frequência em sair de casa, a uma crença mais forte de que o isolamento diminui a propagação do vírus e a uma percepção maior de vulnerabilidade ao vírus e gravidade da doença. A solidão também se associou a uma maior percepção de gravidade e vulnerabilidade à doença, além de uma menor autoconfiança em permanecer em casa. Já a resiliência foi associada a uma menor percepção de vulnerabilidade e gravidade do vírus e a uma maior autoconfiança em permanecer em casa. Por fim, o suporte social esteve associado a uma crença mais forte de que o isolamento diminui a propagação do vírus e a uma maior autoconfiança em realizar o isolamento domiciliar.

Tabela 5

Relações entre crenças/atitudes sobre covid-19/isolamento domiciliar e indicadores de saúde mental

\begin{tabular}{lcccc}
\hline & Resiliência & Suporte Social & Depressão & Solidão \\
\hline Frequência saídas de casa & .092 & -.074 & $-.148^{* *}$ & -.056 \\
Percepção vulnerabilidade & $-.203^{* *}$ & .022 & $.225^{* *}$ & $.122^{*}$ \\
Percepção gravidade & $-.183^{* *}$ & -.039 & $.242^{* *}$ & $.157^{* *}$ \\
Isolamento diminui propagação & -.028 & $.109^{*}$ & $.114^{*}$ & .060 \\
Isolamento evita contaminação & -.036 & .096 & .072 & .073 \\
Autoconfiança para isolamento domiciliar & $.132^{* *}$ & $.240^{* *}$ & -.051 & $-.142^{* *}$ \\
\hline
\end{tabular}

Nota. ${ }^{*} p<.05 ; * * p<.01$ 


\section{Discussão}

Este estudo investigou frequências e relações entre crenças, atitudes e alguns indicadores de saúde mental (depressão, solidão, resiliência e suporte social), no contexto da pandemia da covid-19, em amostra de idosos brasileiros que utilizam a Internet. De forma geral, observou-se que $15,1 \%$ da amostra deste estudo apresentou triagem positiva para depressão. A autoconfiança para realizar isolamento domiciliar relacionou-se significativamente com menor frequência de saídas de casa e melhores índices de saúde mental, ao passo que piores índices de saúde mental associaram-se a uma percepção mais forte sobre a ameaça da covid-19 à saúde. Desta forma, a autoconfiança em realizar isolamento domiciliar e as crenças sobre a gravidade e vulnerabilidade à doença parecem ser componentes psicológicos relevantes de serem trabalhados em intervenções com idosos para promoção da saúde mental e maior adesão ao isolamento domiciliar, no contexto de pandemia da covid-19.

No entanto, o perfil sociodemográfico da amostra não foi representativo da população idosa brasileira. São idosos mais jovens, bastante escolarizados, com acesso e conhecimento para uso da Internet, provenientes do Sudeste, uma região brasileira mais privilegiada em termos socioeconômicos. Logo, trata-se de uma amostra com condições sociodemográficas e econômicas mais favoráveis, que devem ser levadas em conta para a interpretação do perfil predominante em saúde mental observado: médias que apontaram para índices mais altos de resiliência e suporte social, bem como índices mais baixos de depressão e solidão. Foi constatado também que $15,1 \%$ da amostra apresentou triagem positiva para depressão.

Um estudo chinês (Huang \& Zhao, 2020) realizado a partir de uma survey on-line, com 7.236 respondentes, identificou prevalência de sintomas de depressão igual a 20,1\% na população geral, além de ter constatado que as pessoas mais jovens (com menos de 35 anos) reportaram significativamente mais sintomas de depressão do que pessoas mais velhas (com mais de 35 anos). Outra survey on-line conduzida no Brasil com 1.460 participantes com média de idade igual a 32,9 anos identificou também que pessoas com mais idade apresentaram menor risco para desenvolver sintomas de depressão, comparados aos indivíduos mais jovens (Filgueiras \& Stults-Kolehmainen, 2020). Pode-se levantar a hipótese de que a idade seja um fator protetivo para a saúde mental no contexto da pandemia, talvez porque os idosos que participaram de pesquisas on-line são, em sua maioria, aposentados e altamente escolarizados, e por isso não teriam de lidar com mudanças na rotina de trabalho, medo de desemprego, insegurança financeira e pobreza, que são graves consequências econômicas que afetam seriamente a saúde mental das pessoas atuantes no mercado de trabalho (Frasquilho et al., 2016). Entretanto, outro estudo na China, de base populacional, apontou que pessoas com mais de 60 anos apresentaram maiores pontuações para sintomas de depressão, ansiedade e estresse, justamente por fazerem parte de grupo de risco com maiores taxas de mortalidade ante a covid-19 (Qiu et al., 2020).

No entanto, torna-se difícil estabelecer comparações das médias e frequências dos indicadores de saúde mental encontrados no presente estudo com outros que também investigaram temáticas semelhantes, uma vez que a metodologia empregada nesta pesquisa (uso de amostra de conveniência via surveys on-line) não permite inferir dados epidemiológicos representativos. Mesmo outras surveys on-line realizadas em outros contextos apresentam uma série de limitações, pois suas inferências são baseadas em dados de pessoas mais 
propensas a se voluntariar para pesquisa e com acesso à Internet, deixando de fora uma parcela significativa de pessoas sem acesso à Internet ou menos predispostas a participar de pesquisas on-line, sendo o caso de pessoas acima de 75 anos e também indivíduos que sofrem de transtornos mentais graves, que são grupos mais vulneráveis (Pierce et al., 2020). Desta forma, os resultados com relação à frequência e aos índices dos indicadores de saúde mental encontrados neste estudo são úteis apenas para fornecer insights iniciais sobre o perfil de saúde mental de idosos no contexto da pandemia da covid-19 no Brasil, mas certamente não refletem a realidade da maioria dos idosos brasileiros.

Foi constatado que $87,6 \%$ dos idosos relataram ficar em casa ou sair até cinco vezes num período de duas semanas. A autoconfiança para realizar o isolamento domiciliar, bem como as crenças de que o isolamento domiciliar ajuda a evitar a propagação e a contaminação pelo coronavírus, foi bastante frequente entre os participantes, seguida da percepção de gravidade da doença e, depois, da percepção de vulnerabilidade ao vírus, esta última menos frequente em relação às demais crenças. De forma geral, a maioria dos idosos participantes deste estudo relatou estar seguindo a recomendação de permanecer em casa ou sair o mínimo possível, bem como informou estar consciente da gravidade da doença e da importância de seguir as medidas de restrição social, para mitigar os efeitos do vírus. Essa maior consciência das implicações da doença e a maior adesão às medidas de proteção podem estar relacionadas à alta escolaridade observada na amostra, uma vez que idosos com maior instrução têm mais acesso a informações e serviços de saúde (Lima-Costa, 2004), o que pode contribuir para o desenvolvimento de crenças e atitudes favoráveis à saúde.

Quando examinadas as relações entre as crenças e atitudes sobre a covid-19 e o isolamento domiciliar, os resultados oferecem suporte ao MCS (Rosenstock et al., 1988,1994; Borges \& Barletta, 2015), uma vez que foram identificadas associações significativas entre todos os componentes psicológicos do modelo examinado neste estudo, exceto relações entre vulnerabilidade percebida com frequência das saídas de casa e autoconfiança para realizar o isolamento. Logo, os dados deste estudo demonstraram que perceber-se vulnerável à covid-19 não apresenta relação direta com a frequência com que o idoso sai de casa ou com a capacidade que o idoso sente em realizar o isolamento domiciliar.

O MCS postula que a combinação da gravidade e vulnerabilidade percebida constitui a ameaça da doença percebida, e que este componente influenciaria de forma mais direta o comportamento preventivo à saúde (Borges \& Barletta, 2015). Desta forma, pode ser que apenas a percepção de vulnerabilidade em si não seja o suficiente para determinar a adesão ou não ao isolamento, ou mesmo a autoconfiança para adotar esta medida, o que pode explicar a não associação entre vulnerabilidade percebida com frequência em sair de casa e autoconfiança para realizar isolamento domiciliar.

A ameaça percebida não foi diretamente aferida neste estudo, mas foi constatada relação significativa de magnitude moderada entre vulnerabilidade e gravidade percebida, o que oferece suporte para a hipótese de que a ameaça percebida seria uma combinação entre esses dois construtos. Um estudo que envolva modelagem de equações estruturais pode ajudar a elucidar melhor como os componentes psicológicos do MCS se influenciam e se combinam para explicar a adesão ou não ao isolamento domiciliar, embora já haja evidências de que quase todos esses componentes apresentam relações diretas e significativas entre si. 
Analisar as crenças e atitudes dos idosos no contexto da pandemia da covid-19, tendo o MCS como referencial teórico, é útil para se pensar em estratégias interventivas que visam atuar em crenças e atitudes dos indivíduos, com o objetivo final de aumentar a probabilidade de emissão do comportamento preventivo à saúde desejável, no caso, o isolamento domiciliar. $\mathrm{O}$ estudo demonstrou que a frequência de saídas de casa relacionou-se de forma significativa e moderada à percepção de capacidade em realizar o isolamento domiciliar. A autoconfiança apresentou também associações significativas moderadas com os benefícios percebidos do isolamento domiciliar (diminuição da propagação e contaminação pelo coronavírus). Desta forma, a autoconfiança do idoso em realizar o isolamento domiciliar parece ser um componente psicológico central para ser trabalhado com idosos no contexto da pandemia, visando aumentar as chances de que o idoso permaneça em casa com maior frequência, bem como perceba as vantagens de aderir a essa prática.

A autoconfiança para aderir ao isolamento domiciliar foi também associada a um meIhor quadro de saúde mental (mais resiliência, mais suporte social e menos solidão). Idosos mais resilientes, com mais suporte social e que experimentavam menos sintomas de solidão também foram os que apresentaram maior capacidade de permanecer em casa. O desenvolvimento de crenças favoráveis à adesão de práticas recomendadas de saúde parece ser uma ação necessária não apenas para aumentar as chances de adesão ao isolamento, mas também de garantir melhores níveis de saúde mental aos idosos em tempos de pandemia. Intervenções que se baseiam no fortalecimento e desenvolvimento de crenças positivas associadas a comportamentos de saúde, bem como outros construtos que remetem a virtudes e potencialidades humanas (tais como resiliência e suporte social), parecem ser bastante promissoras neste contexto de pandemia (Zanon, Dellazzana-Zanon, Wechsler, Fabretti, \& Rocha, 2020), inclusive para idosos.

O isolamento social pode impactar negativamente a saúde mental de idosos, uma vez que este público já é mais propenso a desenvolver sintomas de solidão e depressão (Armitage \& Nellums, 2020). De fato, este estudo demonstrou que os idosos que estão saindo menos de casa também apresentam maiores chances para desenvolver depressão. No entanto, a depressão apresentou associações de magnitudes mais fortes com a ameaça percebida da doença (percepção de vulnerabilidade e gravidade), sugerindo que o desenvolvimento de depressão em idosos em tempos de pandemia estaria mais fortemente associado às crenças sobre a gravidade e vulnerabilidade à doença do que à restrição social. A percepção da ameaça da doença (combinação da gravidade e vulnerabilidade percebidas) associou-se também a menores níveis de resiliência e a mais sintomas de solidão. Logo, estar consciente da ameaça da doença a sua própria saúde tem mais relação com o estado psicológico dos idosos do que ter de diminuir a frequência das saídas de casa. Pode ser que crenças mais fortes sobre a gravidade da doença e percepção de vulnerabilidade estejam também ligadas a uma exposição exagerada de informações sobre a situação relacionada à pandemia, que, por sua vez, apareceram associadas a maiores índices de ansiedade, estresse e depressão em estudos brasileiros e chineses (Filgueiras \& Stults-Kolehmainem, 2020; Huang \& Zhao, 2020). Embora seja importante que as pessoas estejam conscientes do risco e da gravidade da situação relacionada à pandemia da covid-19, este estudo sugere que uma percepção mais intensa acerca dos riscos e da gravidade da situação associa-se a uma pior saúde mental. 
Este estudo apresenta uma série de limitações. Talvez a maior delas seja o fato de a amostra ser de conveniência e ter excluído idosos sem acesso à Internet. Embora existam dados que apontem que o acesso à Internet vem aumentando entre os idosos brasileiros (Batista, Souza, Schwartz, Exner, \& Almeida, 2015), ainda, uma pequena parcela desta população usa dispositivos tecnológicos para acessar Internet (Sousa, Lima, Cesar, \& Barros, 2018). De fato, contatar esta população tanto para obter dados de pesquisa quanto para direcionar serviços de saúde mental no contexto de pandemia se tornou um grande desafio, justamente por se tratar de um público com contato bastante limitado com dispositivos tecnológicos e Internet, o qual deve ainda permanecer isolado por ser considerado grupo de risco (Yang et al., 2020). Logo, o acesso a informações que tragam dados acurados sobre a saúde mental desses indivíduos fica também bastante dificultado. Dada a assistência insuficiente a este grupo vulnerável, autoridades e profissionais deveriam unir esforços para o estabelecimento de ações que deem conta de superar essas barreiras de alcance, possibilitando o acesso a dados representativos e tornando viáveis a implementação e a execução de ações que visem promover a saúde mental neste contexto de crise.

Ainda, é necessário a investigação de outros indicadores de saúde mental em idosos brasileiros, tais como ansiedade e estresse, não acessados neste estudo. Por outro lado, a investigação de construtos positivos, tais como otimismo, esperança, prática de atividades prazerosas etc., é relevante também, uma vez que intervenções pautadas pela Psicologia Positiva parecem ser bastante promissoras no contexto da pandemia (Zanon et al., 2020).

Este estudo trouxe informações iniciais a respeito da frequência de crenças sobre a pandemia da covid-19 e do perfil de saúde mental de idosos brasileiros com melhores condições sociodemográficas. A autoconfiança para realizar o isolamento domiciliar parece ser um componente psicológico central a ser trabalhado em intervenções que visem garantir maior adesão desta população ao isolamento domiciliar e em intervenções que visem melhorar o quadro de saúde mental de idosos. Um pior quadro de saúde mental foi associado a uma percepção mais forte sobre a ameaça da covid-19 à saúde, sugerindo que crenças sobre a gravidade e vulnerabilidade à doença também devem ser foco de intervenções que visem promover a saúde mental de idosos no contexto de crise gerado pela pandemia. Embora este estudo traga dados que possam ser úteis para a formulação de ações de proteção à saúde mental de idosos em tempos de pandemia, estudos com amostras representativas da população idosa brasileira para mapear perfil de saúde mental são urgentes, uma vez que os idosos constituem importante grupo de risco para a covid-19.

\section{Referências}

Armitage, R., \& Nellums, L. B. (2020). COVID-19 and the consequences of isolating the elderly. Lancet Public Health. doi:http://dx.doi.org/10.1016/S2468-2667(20)30061-X

Barroso, S. M., Andrade, V. S., Midgett, A. H., \& Carvalho, R. G. N. (2016). Evidências de validade da Escala Brasileira de Solidão UCLA. Jornal Brasileiro de Psiquiatria, 65(1), 6875. doi:http://dx.doi.org/10.1590/0047-2085000000105

Batista,M.P.P.,Souza, F.G.,Schwartz,G., Exner,C., \&Almeida,M.H.M.(2015).Utilizaçãonocotidiano de tecnologias da informação e comunicação por idosos participantes da Universidade Aberta da Terceira Idade da Universidade de São Paulo. Revista Kairós - Gerontologia, 18(4), 405-426. doi:http://dx.doi.org/10.23925/2176-901X.2015v18i4p405-426 
Borges, L. M., \& Barletta, J. B. (2015). Teorias e modelos explicativos em prevenção e promoção da saúde orientados para o indivíduo e para a comunidade. In S. G. Murta, C. Leandro-França, K. B. dos Santos, Polejack, L. (Orgs), Prevenção e promoção em saúde mental: Fundamentos, planejamento e estratégias de intervenção (Cap. 5, pp. 113-149). Novo Hamburgo: Sinopsys.

Brasil. (2020). Ministério da Saúde. Protocolo de Manejo Clínico do Coronavírus (Covid-19) na atenção primária à saúde. Recuperado de https://www.saude.gov.br/images/pdf/2020/ marco/20/20200318-ProtocoloManejo-ver002.pdf

Chagas, M. H. N., Crippa, J. A. S., Loureiro, S. R., Hallak, J. E. C., Meneses-Gaya, C. de, Machado-de-Sousa, J. P., . . Tumas, V. (2011). Validity of the PHQ-2 for the screening of major depression in Parkinson's disease: Two questions and one important answer. Aging \& Mental Health, 15(7), 838-843. doi:https://doi.org/10.1080/13607863.2011.569482

Deng, S. Q., \& Peng, H. J. (2020). Characteristics of and Public health responses to the coronavirus disease 2019 outbreak in China. Journal of Clinical Medicine, 9(575), 1-10. doi:http://dx.doi.org/10.3390/jcm9020575

Filgueiras, A., \& Stults-Kolehmainen, M. (2020). The relationship between behavioural and psychosocial factors among Brazilians in quarantine due to COVID-19. SSRN Electronic Journals: The English \& Commonwealth Law Abstracts Journal. doi:http://dx.doi. org/10.2139/ssrn.3566245

Frasquilho, D., Matos, M. G., Salonna, F., Guerreiro, D., Storti, C. C., Gaspar, T., Caldasde-Almeida, J. M. (2016). Mental health outcomes in times of economic recession: A systematic literature review. BMC Public Health, 16(115), 1-40. doi:http://dx.doi. org/10.1186/s12889-016-2720-y

Gaya, C. M. (2011). Estudo de validação de instrumentos de rastreamento para transtornos depressivos, abuso e dependência de álcool e tabaco (Tese, Universidade de São Paulo, Ribeirão Preto, 238 p.)

Huang, Y., \& Zhao, N. (2020). Generalized anxiety disorder, depressive symptoms and sleep quality during COVID-19 outbreak in China: A web-based cross-sectional survey. Psychiatry Research, 288, 112954. doi:http://dx.doi.org/10.1016/j.psychres.2020.112954

Lima-Costa, M. F. (2004). Influência da idade e da escolaridade no uso de serviços preventivos de saúde - Inquérito de Saúde da Região Metropolitana de Belo Horizonte, Minas Gerais, Brasil. Epidemiologia e Serviços de Saúde, 13(4). 209-215. doi:http://dx.doi.org/10.5123/ S1679-49742004000400003

Liu, K., Chen, Y., Lin, R. \& Han, K. (2020). Clinical feature of COVID-19 in elderly patients: A comparison with young and middle-aged patients. Journal of Infection. 80(6), e14-e18. doi:https://doi.org/10.1016/j.jinf.2020.03.005

Neri, A. L., \& Vieira, L. A. M. (2013). Envolvimento social e suporte social percebido na velhice. Revista Brasileira de Geriatria e Gerontologia, 16(3), 419-432. doi:http://dx.doi. org/10.1590/S1809-98232013000300002

Osório, F. L., Mendes, A. V., Crippa, J. A., \& Loureiro, S. R. (2009). Study of the discriminative validity of the PHQ-9 and PHQ-2 in a sample of Brazilian women in the context of primary health care. Perspectives in Psychiatric Care, 45, 216-227. doi:http://dx.doi. org/10.1111/j.1744-6163.2009.00224.x 
Pierce, M., McManus, S., Jessop, C., John, A., Hotopf, M., Ford, T., ... Abel, K. M. (2020). Says who? The significance of sampling in mental health surveys during COVID-19. The Lancet Psychiatry, 7(7), 567-568. doi: https://doi.org/10.1016/ S2215-0366(20)30237-6

Qiu, J., Shen, B., Zhao, M., Wang, Z., Xie, B. \& Xu, Y. (2020). A nationwide survey of psychological distress among Chinese people in the COVID-19 epidemic: Implications and policy recommendations. General Psychiatry, 33, e100213corr1. doi:http://dx.doi. org/10.1136/gpsych-2020-100213corr1

Rosenstock, I. M., Stretcher, V. I., \& Becker, M. H. (1988). Social learning theory and the Health Belief Model. Health Education Quarterly, 15(2), 175-183. doi:http://dx.doi. org/10.1177/109019818801500203

Rosenstock, I. M., Stretcher, V. I., \& Becker, M. H. (1994). The Health Belief Model and the HIV risk behavior change. In R. J. DiClemente, \& J. L. Peterson (Ed.), Preventing AIDS: Theories and methods of behavioural interventions (pp. 5-24). New York: Plenum Press.

Russell, D.; Peplau, L. A., \& Cutrona, C. E. (1980), The revised UCLA Loneliness Scale: Concurrent and discriminant validity evidence. Journal of Personality and Social Psychology, 39(3), 472-480. doi:https://doi.org/10.1037/0022-3514.39.3.472

Sousa, N. F. S., Lima, M. G., Cesar, C. L. G., \& Barros, M. B. A. (2018). Envelhecimento ativo: Prevalênciae diferenças degêneroeidadeemestudo debasepopulacional.CadernodeSaúde Pública, 34(11), e00173317, 1-14. doi:https://dx.doi.org/10.1590/0102-311x00173317

Smith, B. W., Dalen, J., Wiggins, K., Tooley, E., Christopher, P., \& Bernard, J. (2008). The Brief Resilience Scale: Assessing the Ability to Bounce Back. International Journal of Behavioral Medicine, 15(3), 194-200. doi:http://dx.doi.org/10.1080/10705500802222972

Wilder-Smith, A., \& Freedman, D. O. (2020). Isolation, quarantine, social distancing and community containment: Pivotal role for old-style public health measures in the novel coronavirus (2019-nCoV) outbreak. Journal of Travel Medicine,27(2), 1-4. doi:http:// dx.doi.org/10.1093/jtm/taaa020

World Health Organization. (2020a). Coronavirus disease 2019 (COVID-19): Situation report 51. World Health Organization. Recuperado de https://www.who.int/docs/default-source/ coronaviruse/situation-reports/20200311-sitrep-51-covid-19.pdf?sfvrsn=1ba62e57_10

World Health Organization. (2020b). Coronavirus disease 2019 (COVID-19): Situation report - 143. World Health Organization. Recuperado de https://www.who.int/ docs/default-source/coronaviruse/situation-reports/20200611-covid-19-sitrep-143. pdf?sfvrsn=2adbe568_4

Yang, Y., Li, W., Zhang, Q., Zhang, L., Cheung, T. \& Xiang, Y. T. (2020). Mental health services for older adults in China during the COVID-19 outbreak. The Lancet, 7(4), e19. doi:https:// doi.org/10.1016/S2215-0366(20)30079-1

Zanon, C., Dellazzana-Zanon, L. L., Wechsler, S. M., Fabretti, R. R., \& Rocha, K. N. (2020). COVID-19: Implicações e aplicações da Psicologia Positiva em tempos de pandemia. Estudos dePsicologia, 37(e200072), 1-13. doi:https://doi.org/10.1590/1982-0275202037e200072

Recebido em: 18/06/2020

Última revisão: 21/08/2020

Aceite final: 06/10/2020 


\section{Sobre a autora:}

Heloísa Gonçalves Ferreira: Doutora pela Universidade Federal de São Carlos (UFSCar). Professora no Departamento de Cognição e Desenvolvimento, Instituto de Psicologia na UFSCar. E-mail: helogf@gmail.com, Orcid: https://orcid.org/0000-0002-3545-9378 
